\title{
Finite-time and fixed-time stabilization for integrator chain of arbitrary order*
}

\author{
Konstantin Zimenko ${ }^{1}$, Andrey Polyakov ${ }^{1,2}$, Denis Efimov ${ }^{1,2}$ and Wilfrid Perruquetti ${ }^{2}$
}

\begin{abstract}
In the present paper, homogeneous control laws are designed for finite-time and fixed-time stabilization of integrator chains of arbitrary order. Provided analysis is based on Lyapunov function method and homogeneity concept. Fixed-time convergence is achieved by use of hybrid control algorithm with homogeneity degree changing. Performance of the resulting finite-time and fixed-time feedbacks is illustrated by numerical simulations.
\end{abstract}

\section{INTRODUCTION}

Motivated by modern control applications, the finite-time and fixed-time stabilization feedback design is continuing to be the subject of numerous studies (see, for example, [1]-[18]). Among these studies and many others, particular attention is paid to finite/fixed-time stabilization of integrator chains as a basic model for demonstration and further extension for multi-input multi-output linear plants. Interest to the disturbed integrator chain control systems is additionally motivated by different mechanical and electromechanical applications (see, for example, [20], [22]). However, most of existing control laws are discontinuous (as, for example, in [6], [7]), designed to stabilize the chain of integrators only of a certain order (for second order [14], [15] for third order [16], [17], etc.), or even depending directly on the initial conditions ([17]).

The present paper is devoted to Lyapunov-based controller design which stabilizes the arbitrary order integrator chain in finite/fixed time. Presented control algorithms are obtained with use of Lyapunov function method and homogeneity concept.

The paper [1] is related to development of robust finitetime and fixed-time stabilization controllers, which depend on implicitly defined homogeneous Lyapunov function. For practical implementation of these control algorithms it is required to use special computational procedures for calculating Implicit Lyapunov Function (ILF). In [11] the finitetime control law has been modified to explicit form, where instead of ILF the homogeneous norm is used. Then the difference between ILF and homogeneous norm is considered as

*This work is supported by the Russian Science Foundation under grant 17-19-01422.

${ }^{1}$ Konstantin Zimenko, Andrey Polyakov, Denis Efimov are with Department of Control Systems and Informatics, ITMO University, 49 Kronverkskiy av., 197101 Saint Petersburg, Russia. (e-mail: kostyazimenko@gmail.com).

${ }^{2}$ Andrey Polyakov, Denis Efimov and Wilfrid Perruquetti are with Non-A INRIA - LNE, Parc Scientifique de la Haute Borne 40, avenue Halley Bat.A, Park Plaza 59650 Villeneuve d'Ascq (e-mail: denis.efimoveinria.fr, andrey.polyakove

inria.fr, Wilfrid.Perruquettieinria.fr). They are also with CRIStAL (UMR-CNRS 9189), Ecole Centrale de Lille, BP 48, Cite Scientifique, 59651 Villeneuve-d'Ascq, France. additional external disturbance, that has led to a more complex analysis and parameters tuning procedure. The present paper provides the analysis based on an explicitly defined Lyapunov function for the similar control law. This analysis allows to get simple parameters tuning procedure and obtain new estimates for settling-time function. It is also shown that fixed-time convergence can be achieved by changing the homogeneity degree in hybrid control algorithm.

The paper is organized in the following way. The problem formulation is presented in Section II. Section III recalls some basics on finite/fixed-time stability and weighted homogeneity. Section IV presents the main result on finitetime and fixed-time controllers design. Simulation results are shown in Section V for performance illustration of proposed control algorithms. Finally, concluding remarks are given in Section VI.

Through the paper the following notation will be used: $\mathbb{R}$ is the set of real numbers; $\mathbb{R}_{+}=\{x \in \mathbb{R}: x>0\}$; $\operatorname{diag}\left\{\lambda_{i}\right\}_{i=1}^{n}$ is a diagonal matrix with elements $\lambda_{i}$; for $P \in$ $\mathbb{R}^{n \times n}$ the relation $P>0(P<0, P \geq 0, P \leq 0)$ means that the symmetric matrix $P=P^{T}$ is positive (negative) definite (semidefinite); a series of integers $1,2, \ldots, n$ is denoted by $\overline{1, n}$.

\section{PROBLEM FORMULATION}

Consider a chain of $n$ integrators

$$
\dot{x}=A x+b u,
$$

where $x \in \mathbb{R}^{n}$ is the state vector, $u \in \mathbb{R}$,

$$
A=\left(\begin{array}{ccccc}
0 & 1 & 0 & \cdots & 0 \\
0 & 0 & 1 & \cdots & 0 \\
\vdots & \vdots & \vdots & \ddots & \vdots \\
0 & 0 & 0 & \cdots & 1 \\
0 & 0 & 0 & \cdots & 0
\end{array}\right) \text { and } b=\left(\begin{array}{c}
0 \\
0 \\
\vdots \\
0 \\
1
\end{array}\right)
$$

The objective of the present paper is to design Lyapunovbased controllers, which stabilize the arbitrary order system (1) to the origin in finite/fixed time.

\section{PRELIMINARIES}

\section{A. Finite-Time and Fixed-Time Stability}

Let us consider the system

$$
\dot{x}=f(t, x), \quad x(0)=x_{0},
$$

where $x \in \mathbb{R}^{n}$ is the state vector, $f \in \mathbb{R}_{+} \times \mathbb{R}^{n} \rightarrow \mathbb{R}^{n}$ is a vector field. If the vector field $f$ is discontinuous with respect to $x$, then the solutions of (2) fall into the area of 
differential inclusions and need to be understood in the sense of Filippov [23]. For any $x_{0} \in \mathbb{R}^{n}$ denote a corresponding solution by $\tilde{X}\left(t, x_{0}\right)$ for the instants $t \geq 0$ for which it exists, $\tilde{X}\left(0, x_{0}\right)=x_{0}$.

Definition 1 [3], [4]

The origin of (2) is said to be globally finite-time stable if it is globally asymptotically stable and any solution $\tilde{X}\left(t, x_{0}\right)$ of (2) reaches the equilibrium point at some finite time moment, i.e., $\tilde{X}\left(t, x_{0}\right)=0, \forall t \geq T\left(x_{0}\right)$, where $T: \mathbb{R}^{n} \rightarrow$ $\mathbb{R}_{+} \cup\{0\}$ is the settling-time function.

\section{Definition 2 [8]}

The origin of (2) is said to be fixed-time stable if it is globally finite-time stable and the settling-time function $T\left(x_{0}\right)$ is bounded, i.e., $\exists T_{\max }>0: T\left(x_{0}\right) \leq T_{\max }, \forall x_{0} \in$ $\mathbb{R}^{n}$.

\section{Definition 3 [8]}

The set $M$ is said to be globally finite-time attractive for (2) if any solution $\tilde{X}\left(t, x_{0}\right)$ of (2) reaches $M$ in some finite time moment $t=T\left(x_{0}\right)$ and remains there $\forall t \geq$ $T\left(x_{0}\right), T: \mathbb{R}^{n} \rightarrow \mathbb{R}_{+} \cup\{0\}$ is the settling-time function.

Definition 4 [8]

The set $M$ is said to be fixed-time attractive for (2) if it is globally finite-time attractive and the settling-time function $T\left(x_{0}\right)$ is globally bounded by some number $T_{\max }>0$.

Theorem 1 [3], [6]

Suppose there exists a positive definite $C^{1}$ function $V$ defined on an open neighborhood of the origin $D \subset \mathbb{R}^{n}$ and real numbers $C>0$ and $\sigma \geq 0$, such that the following condition is true for the system (2)

$$
\dot{V}(x) \leq-C V^{\sigma}(x), \quad x(t) \in D \backslash\{0\} .
$$

Then depending on the value $\sigma$ the origin is stable with different types of convergence:

- if $\sigma=1$, the origin is asymptotically stable;

- if $0 \leq \sigma<1$, the origin is finite-time stable and

$$
T\left(x_{0}\right) \leq \frac{1}{C(1-\sigma)} V_{0}^{1-\sigma},
$$

where $V_{0}=V\left(x_{0}\right)$;

- if $\sigma>1$ the origin is asymptotically stable and, for every $\epsilon$, the set $B(0, \epsilon)=\{x \in D: V(x)<\varepsilon\}$ is fixedtime attractive with

$$
T_{\max }=\frac{1}{C(\sigma-1) \varepsilon^{\sigma-1}} .
$$

If $D=\mathbb{R}^{n}$ and function $V$ is radially unbounded, then the system (2) is globally stable with respect to the equilibrium point.

\section{B. Weighted Homogeneity}

For $r_{i} \in \mathbb{R}_{+}, i=\overline{1, n}, \rho>2 \max _{i} r_{i}$ and $\lambda>0$ define vector of weights $r=\left(r_{1}, \ldots, r_{n}\right)^{T}$, dilation matrix $D_{r}(\lambda)=\operatorname{diag}\left\{\lambda^{r_{i}}\right\}_{i=1}^{n}$ and homogeneous norm

$$
\|x\|_{r}=\left(\sum_{i=1}^{n}\left|x_{i}\right|^{\frac{\rho}{r_{i}}}\right)^{\frac{1}{\rho}} .
$$

Note that under introduced restriction on $\rho$ the homogeneous norm is a continuously differentiable function out of the origin.

\section{Definition 5 [19]}

A function $g: \mathbb{R}^{n} \rightarrow \mathbb{R}$ (vector field $f: \mathbb{R}^{n} \rightarrow \mathbb{R}^{n}$ ) is said to be $r$-homogeneous of degree $d$ if $g\left(D_{r}(\lambda) x\right)=\lambda^{d} g(x)$ $\left(f\left(D_{r}(\lambda) x\right)=\lambda^{d} D_{r}(\lambda) f(x)\right)$ for fixed $r$, all $\lambda>0$ and $x \in \mathbb{R}^{n}$.

Obviously, homogeneous norm is $r$-homogeneous of degree 1. Introduce the following compact set (homogeneous sphere) $\mathbb{S}_{r}=\left\{x \in \mathbb{R}^{n}:\|x\|_{r}=1\right\}$, then for any $x \in \mathbb{R}^{n}$ there is $y \in \mathbb{S}_{r}$ such that $x=D_{r}(\lambda) y$ for $\lambda=\|x\|_{r}$.

\section{Theorem 2 [18]}

Let $f: \mathbb{R}^{n} \rightarrow \mathbb{R}^{n}$ be defined on $\mathbb{R}^{n}$ and be a continuous $r$-homogeneous vector field with degree $d(d<0)$. If the origin of the system

$$
\dot{x}=f(x)
$$

is locally asymptotically stable then it is globally asymptotically stable (globally finite-time stable) and there exists a continuously differentiable Lyapunov function $V$ which is $r$-homogeneous of degree $v>-d$.

By definition of homogeneity there exist constants $c_{1}, c_{2}>$ 0 such that

$$
c_{1}\|x\|_{r}^{v} \leq V(x) \leq c_{2}\|x\|_{r}^{v}
$$

The $r$-homogeneity presented in Definition 5 is introduced for some $r$ and all $\lambda>0$. Restricting the set of admissible values for $\lambda$ the local homogeneity concept has been introduced in [19], [9], [10].

\section{MAIN RESULT}

For $\mu \in(0,1]$ and the vector of weights $r=(l+$ $(n-1) \mu, l+(n-2) \mu, \cdots, l)^{T}, r_{\max }=l+(n-1) \mu$, $r_{\min }=l$ denote the dilation matrix $D_{r}(\lambda)=\operatorname{diag}\left\{\lambda^{r_{i}}\right\}_{i=1}^{n}$ and diagonal matrix $\tilde{H}=\operatorname{diag}\{-m+(n+1-i) \mu\}_{i=1}^{n}$, where $l \in \mathbb{R}_{+}: l \geq \mu, m \in \mathbb{R}_{+}: m>n \mu$.

\section{Theorem 4}

If the system of matrix inequalities:

$$
X>0
$$

$$
\begin{aligned}
& \alpha_{\max }(\tilde{H} X+X \tilde{H})+A X+X A^{T}+b y+y^{T} b^{T} \leq-\gamma X, \\
& -\alpha_{\max }(\tilde{H} X+X \tilde{H})+A X+X A^{T}+b y+y^{T} b^{T} \leq-\gamma X
\end{aligned}
$$

is feasible for some $X \in \mathbb{R}^{n \times n}, y \in \mathbb{R}^{1 \times n}, \gamma \in \mathbb{R}_{+}$and

$$
\begin{aligned}
\alpha_{\max }= & \left(1+\left(\sum\left|k_{i}\right|^{\frac{\rho}{\rho-r_{\min }}}\right)^{\frac{\rho-r_{\min }}{r_{\min }}}\right)^{\frac{r_{\min }}{\rho}} \times \\
& \left(\sum\left(\frac{1}{r_{i}}\right)^{\frac{\rho}{r_{\max }-r_{\min }}}\right)^{\frac{r_{\max }-r_{\min }}{\rho}},
\end{aligned}
$$

$P=X^{-1}, k=y X^{-1}$, then the control of the form

$$
u=\|x\|_{r}^{l-\mu} k D_{r}\left(\frac{1}{\|x\|_{r}}\right) x
$$


stabilizes the origin of the system (1) in a finite time and the settling-time function estimate has the form

$$
T(x) \leq \frac{2(m+l-\mu) V_{0}^{\mu}}{\mu \gamma c_{1}^{\mu}},
$$

where $V_{0}=\left(x_{0}^{T} H\left(\frac{1}{\left\|x_{0}\right\|_{r}}\right) P H\left(\frac{1}{\left\|x_{0}\right\|_{r}}\right) x_{0}\right)^{\frac{1}{2(m+l-\mu)}}$, $H(\lambda)=\operatorname{diag}\left\{\lambda^{-m+(n+1-i) \mu}\right\}_{i=1}^{n}$ and $c_{1}=$ $\min _{\|x\|_{r}=1}\left\{\left(x^{T} P x\right)^{\frac{1}{2(m+l-\mu)}}\right\}$.

Remark 1 The finite-time control law (10) for $l=1$ coincides with the control presented in [11]. In comparison with [11], presented analysis allows to relax obtained LMIs and simplify the procedure of parameters tuning. Also, new estimates for settling-time function is obtained.

Remark 2 Since the inequalities (7), (8) are feasible at least for sufficiently small $\alpha$ (can be achieved by choosing sufficiently big $l \in \mathbb{R}_{+}$) the presented control scheme implies simple control parameters tuning: it requires to solve LMIs (6) and

$$
\begin{gathered}
\tilde{\alpha}(\tilde{H} X+X \tilde{H})+A X+X A^{T}+b y+y^{T} b^{T} \leq-\gamma X, \\
-\tilde{\alpha}(\tilde{H} X+X \tilde{H})+A X+X A^{T}+b y+y^{T} b^{T} \leq-\gamma X,
\end{gathered}
$$

for some $\tilde{\alpha} \in \mathbb{R}_{+}$and then choose such $l$ for which $\alpha_{\max } \leq$ $\tilde{\alpha}$.

Remark 3 The parameter $m$ can be chosen large enough to have $\tilde{H} X+X \tilde{H} \leq 0$. In this case the inequality (7) can be reduced.

Remark 4 Parameters $l, m, \mu$ and $\gamma$ allows the upper bound of the settling time function (11) to be adjusted.

Remark 5 For $l=\mu \leq 1$ the control (10) is continuous outside the origin and globally bounded for all $x \in \mathbb{R}^{n}$ :

$$
\begin{aligned}
& u^{2}=x^{T} D_{r}\left(\frac{1}{\|x\|_{r}}\right) k^{T} k D_{r}\left(\frac{1}{\|x\|_{r}}\right) x \leq \\
& \varsigma\|x\|_{r}^{-2 m} x^{T} H\left(\frac{1}{\|x\|_{r}}\right) P H\left(\frac{1}{\|x\|_{r}}\right) x= \\
& \varsigma\|x\|_{r}^{-2 m} V^{2 m} \leq \varsigma c_{2}^{2 m},
\end{aligned}
$$

where $\varsigma \in \mathbb{R}_{+}: k^{T} k \leq \varsigma P$.

For $\mu<\min \{l, 1\}$ the control (10) is continuous in the state variable $x$. If $\mu \rightarrow 0$ then the feedback (10) becomes a linear $u=k x$. If $\mu=l \leq 1$ the control is discontinuous at the origin and continuous outside. For practical realization of the control, the discontinuous feedback law can be replaced with a high-gain linear feedback if the system state is close to the origin as in [1].

Remark 6 It can be easily checked that the system (1), (10) is homogeneous of degree $-\mu$. Thus, the system has such qualitative stability properties of homogeneous systems as Input-to-State Stability with respect to additive perturbations in the right-hand side of (1) and measurement noises in the feedback.

Remark 7 The controller (10) can also be treated as the weighted homogeneous version of the unit sliding mode control [20], [21].

Note that in presented control scheme instead of homogeneous norm (3) another homogeneous function of degree 1 can be used. In this case the main difference is in calculating the parameter $\alpha$. Also if we choose instead of homogeneous norm implicitly defined homogeneous function equal to $V$, then for $m=l=1$ we obtain implicitly defined Lyapunov function

$$
Q=x^{T} D_{r}\left(V^{-1}\right) P D_{r}\left(V^{-1}\right) x-1
$$

and control $u=V^{1-\mu} k D_{r}\left(V^{-1}\right) x, V \in \mathbb{R}_{+}: Q(V, x)=0$ as in [1], [2].

Convergence within a fixed interval of time irrespectively of its initial conditions can be achieved by changing the degree of homogeneity in hybrid control algorithm. The following discontinuous fixed-time controller is based on time dependent switching scheme for the first time presented in [24] for an observer.

In this case for $m_{1}, m_{2} \in \mathbb{R}_{+}: m_{1}=m_{2}+2 \mu, m_{1}>n \mu$, $l>(n-1) \mu, \mu \in(0,1]$ denote vectors of weights $r_{1}=$ $(l+(n-1) \mu, l+(n-2) \mu, \cdots, l)^{T}, r_{2}=(l-(n-1) \mu, l-$ $(n-2) \mu, \cdots, l)^{T}$, homogeneous norms $\|x\|_{r_{1}},\|x\|_{r_{2}}$ and matrices $D_{1}(\lambda)=\operatorname{diag}\left\{\lambda^{r_{1}(i)}\right\}_{i=1}^{n}, \tilde{H}_{1}=\operatorname{diag}\left\{-m_{1}+(n+\right.$ $1-i) \mu\}_{i=1}^{n}, D_{2}(\lambda)=\operatorname{diag}\left\{\lambda^{r_{2}(i)}\right\}_{i=1}^{n}, \tilde{H}_{2}=\operatorname{diag}\left\{-m_{2}-\right.$ $(n+1-i) \mu\}_{i=1}^{n}, H_{1}(\lambda)=\operatorname{diag}\left\{\lambda^{-m_{1}+(n+1-i) \mu}\right\}_{i=1}^{n}$, $H_{2}(\lambda)=\operatorname{diag}\left\{\lambda^{-m_{2}-(n+1-i) \mu}\right\}_{i=1}^{n}$.

Define homogeneous functions of degree 1 :

$$
\begin{aligned}
& V_{1}(x)=\left(x^{T} H_{1}\left(\frac{1}{\|x\|_{r_{1}}}\right) P H_{1}\left(\frac{1}{\|x\|_{r_{1}}}\right) x\right)^{\frac{1}{2\left(m_{1}+l-\mu\right)}}, \\
& V_{2}(x)=\left(x^{T} H_{2}\left(\frac{1}{\|x\|_{r_{2}}}\right) P H_{2}\left(\frac{1}{\|x\|_{r_{2}}}\right) x\right)^{\frac{1}{2\left(m_{2}+l+\mu\right)}} .
\end{aligned}
$$

For $V_{1}$ and $V_{2}$ according to (5) define $c_{1_{1}}, c_{2_{1}}$ and $c_{1_{2}}, c_{2_{2}}$ correspondingly.

Theorem 5

If the system of matrix inequalities (6),

$$
\begin{array}{r}
\alpha_{1}\left(\tilde{H}_{1} X+X \tilde{H}_{1}\right)+A X+X A^{T}+b y+y^{T} b^{T} \leq-\gamma_{1} X, \\
-\alpha_{1}\left(\tilde{H}_{1} X+X \tilde{H}_{1}\right)+A X+X A^{T}+b y+y^{T} b^{T} \leq-\gamma_{1} X, \\
\alpha_{2}\left(\tilde{H}_{2} X+X \tilde{H}_{2}\right)+A X+X A^{T}+b y+y^{T} b^{T} \leq-\gamma_{2} X, \\
-\alpha_{2}\left(\tilde{H}_{2} X+X \tilde{H}_{2}\right)+A X+X A^{T}+b y+y^{T} b^{T} \leq-\gamma_{2} X,
\end{array}
$$

is feasible for some $X \in \mathbb{R}^{n \times n}, y \in \mathbb{R}^{1 \times n}, \gamma_{1}, \gamma_{2} \in \mathbb{R}_{+}$, were $\alpha_{1}, \alpha_{2}$ are calculated according to (9) with $r_{1}$ and $r_{2}$ correspondingly, $P=X^{-1}, k=y X^{-1}$, then the control of the form

$$
u=\left\{\begin{array}{l}
\|x\|_{r_{2}}^{l+\mu} k D_{2}\left(\frac{1}{\|x\|_{r_{2}}}\right) x \text { for } t \leq T_{s}, \\
\|x\|_{r_{1}}^{l-\mu} k D_{1}\left(\frac{1}{\|x\|_{r_{1}}}\right) x \text { for } t>T_{s},
\end{array}\right.
$$

for some $T_{s} \in \mathbb{R}_{+}$stabilizes the origin of the system (1) in a fixed time

$$
T(x) \leq 2\left(m_{1}+l-\mu\right) \mu^{-1}\left(\frac{c_{2_{2}}^{\mu}}{\gamma_{2} E^{\mu}}+\frac{B^{\mu}}{\gamma_{1} c_{1_{1}}^{\mu}}\right),
$$

where $E=\left(\frac{2\left(m_{1}+l-\mu\right) c_{22}^{\mu}}{\mu \gamma_{2} T_{s}}\right)^{\frac{1}{\mu}}$,

$$
B=\max _{V_{2}=E} V_{1} .
$$


Note, that the parameter $T_{s} \in \mathbb{R}_{+}$can be arbitrarily chosen.

Similarly to finite-time case, remarks analogous to 2-6 can be presented for fixed-time control algorithm. For example, the fixed-time controller is constructed by switching homogeneity properties of the system: it is homogeneous with positive degree $\mu$ for $t \leq T_{s}$ and with negative degree $-\mu$ for $t>T_{s}$.

The convergence rate of proposed control algorithms can be accelerated via time-rescaling in similar way, as in [12], [6], [13].

Remark 8 For $\lambda>1$ and $N=\operatorname{diag}\left\{\lambda^{1-i}\right\}_{i=1}^{n}$ rewrite the control (16) (or control (10)) as $\bar{u}(x)=\lambda^{n} u(N x)$. Then for $\bar{x}=N x, \bar{t}=\lambda t, \bar{T}_{s}=T_{s} / \lambda$ we obtain $\dot{\bar{x}}=$ $A \bar{x}(\bar{t})+b u(\bar{x}(\bar{t}))$ and the following estimates of settlingtime function: $T\left(x_{0}\right) \leq \frac{T_{1}+T_{s}}{\lambda}$ for the control (16) and $T\left(x_{0}\right) \leq \frac{2(m+l-\mu) V\left(\bar{x}_{0}\right)^{\mu}}{\mu \gamma c_{1}^{\mu} \lambda}$ for the control (10).

\section{SIMULATION RESULTS}

Let us demonstrate the performance of presented control laws using the system of triple integrator with initial conditions $x_{1}(0)=10, x_{2}(0)=-30, x_{3}(0)=0$. Using Theorem 4 the finite-time stabilizing controller is designed for this system in the form (10) with the parameters $l=20$, $m=1, \mu=0.3, \rho=41.201$, where the matrix $P \in \mathbb{R}^{3 \times 3}$, $P>0$ and the vector $k \in \mathbb{R}^{1 \times 3}$ are obtained from the inequalities (6)-(8):

$$
\begin{gathered}
P=\left(\begin{array}{ccc}
23.5107 & 16.5213 & 2.9767 \\
16.5213 & 14.6850 & 3.0667 \\
2.9767 & 3.0667 & 1.0731
\end{array}\right), \\
k=\left(\begin{array}{lll}
-7.0626 & -10.5577 & -3.9899
\end{array}\right) .
\end{gathered}
$$

The parameters of the fixed-time stabilizing controller (16) were selected solving the LMI (6), (12)-(15) with the parameters $l=40, m_{1}=0.91, m_{2}=0.31, \mu=0.3$, $\rho_{1}=\rho_{2}=81.7$ :

$$
\begin{gathered}
P=\left(\begin{array}{lll}
0.0235 & 0.0217 & 0.0064 \\
0.0217 & 0.0287 & 0.0082 \\
0.0064 & 0.0082 & 0.0045
\end{array}\right), \\
k=\left(\begin{array}{lll}
-20.2539 & -21.1388 & -6.8414
\end{array}\right) .
\end{gathered}
$$

The parameter $T_{s}$ has been chosen equal to 1.5.

Simulations for finite-time and fixed-time convergence accelerated with $\lambda=2$ have also been carried out. All simulations results are presented in Fig.1-6.

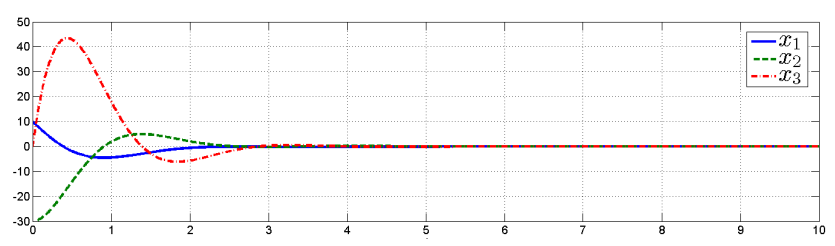

Fig. 1. System states versus time for finite-time control

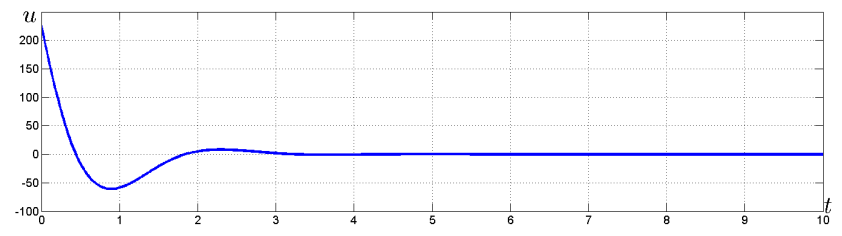

Fig. 2. Control law (10) versus time

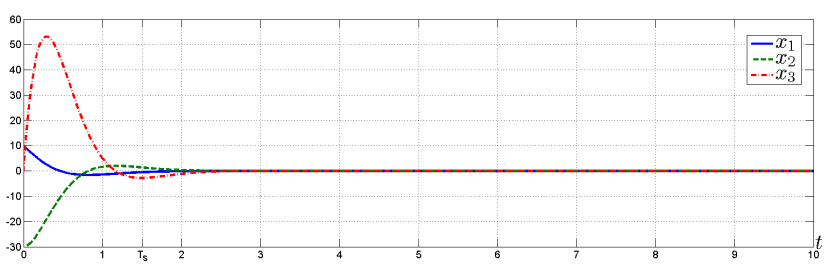

Fig. 3. System states versus time for fixed-time control

\section{CONCLUSIONS}

The paper is focused on finite-time and fixed-time control for integrator chains of arbitrary order. Obtained finite-time control design method consist in appropriate modification of control law presented in [11] and use of analysis based on explicitly defined Lyapunov function. Based on this result simple procedure of parameters tuning and new estimates for settling-time function were obtained. The fixed-time control algorithm for chain of integrators is presented. It is shown that fixed-time convergence can be achieved by changing the homogeneity degree in hybrid control algorithm. The performance of obtained control algorithms is demonstrated through simulations.

A detailed study of the presented control algorithms on robustness analysis with respect to disturbances, uncertainties, delays and extension of these results on wider class of systems goes beyond the scope of the paper providing the subject for a future research.

\section{REFERENCES}

[1] A. Polyakov, D. Efimov, W. Perruquetti, Finite-time and fixed-time stabilization: Implicit Lyapunov function approach, Automatica, vol. 51, pp. 332-340, 2015.

[2] A. Polyakov, D. Efimov, W. Perruquetti, Robust Stabilization of MIMO Systems in Finite/Fixed Time, Int. J. Robust. Nonlinear Control, vol.26, pp. 69-90, 2016.

[3] S. Bhat, D. Bernstein, Finite-time stability of continuous autonomous systems, SIAM Journal of Control and Optimization, vol. 38(3), pp. 751-766, 2000.

[4] Y. Orlov, Finite Time Stability and Robust Control Synthesis of Uncertain Switched Systems, SIAM Journal of Control and Optimization, vol. 43(4), pp. 1253-1271, 2004.

[5] J. D. Sánchez-Torres, E. N. Sanchez, A. G. Loukianov, Predefinedtime stability of dynamical systems with sliding modes, in American Control Conference (ACC), pp. 5842-5846, 2015.

[6] S. Laghrouche, M. Harmouche, Y. Chitour, Stabilization of perturbed integrator chains using Lyapunov-Based Homogeneous Controllers, International Journal of Control, pp. 1-10, 2016.

[7] E. Cruz-Zavala, J. A. Moreno, Homogeneous High Order Sliding Mode design: A Lyapunov approach, Automatica, vol. 80, pp. 232238, 2017.

[8] A. Polyakov, Nonlinear feedback design for fixed-time stabilization of linear control systems, IEEE Transactions on Automatic Control, vol. 57, no. 8, pp. 2106 2110, 2012. 


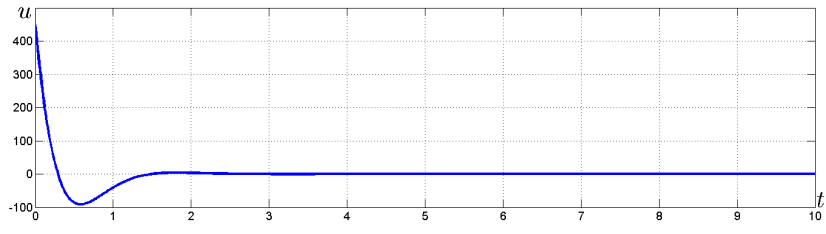

Fig. 4. Control law (16) versus time

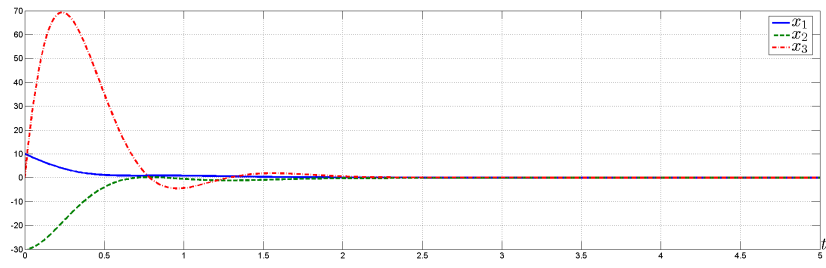

Fig. 5. System states versus time for accelerated finite-time control

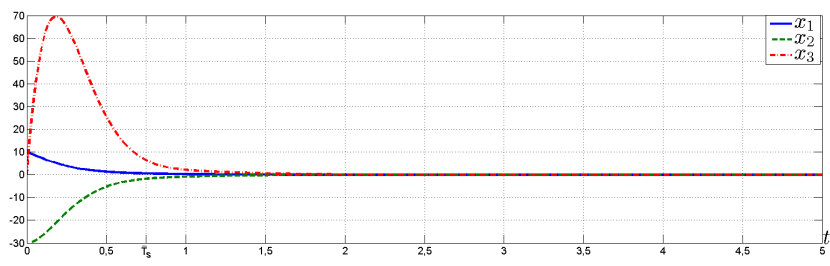

Fig. 6. System states versus time for accelerated fixed-time control

[9] V. Andrieu, L. Praly, A. Astolfi, Homogeneous approximation, recursive observer and output feedback, SIAM Journal of Control and Optimization, vol. 47(4), pp. 1814-1850, 2008.

[10] E. Bernuau, A. Polyakov, D. Efimov, W. Perruquetti, Verification of ISS, iISS and IOSS properties applying weighted homogeneity, System and Control Letters, vol. 62, no. 12, pp. 1159-1167, 2013.
[11] K. Zimenko, A. Polyakov, D. Efimov, Stabilization of Chain of Integrators with Arbitrary Order in Finite-time, in 54th IEEE Conference on Decision and Control, pp. 4637-4641, 2016.

[12] D. Efimov, A. Levant, A. Polyakov, W. Perruquetti, Supervisory Acceleration of Convergence for Homogeneous Systems, International Journal of Control, pp. 1-11, 2017.

[13] A. Levant, Y. Dvir, Accelerated high-order MIMO sliding mode control, In Proc. of the 13th International Workshop on Variable Structure Systems, pp. 1-6, 2014.

[14] Y. Orlov, Y. Aoustin, C. Chevallereau, Finite Time Stabilization of a Perturbed Double Integrator-Part I: Continuous Sliding Mode-Based Output Feedback Synthesis, IEEE Transactions on Automatic Control, vol. 56(3), pp. 614-618, 2011.

[15] Y. Sua, C. Zheng, Robust finite-time output feedback control of perturbed double integrator, Automatica, vol. 60, pp. 86-91, 2015.

[16] P. Trivedi, B. Bandyopadhyay, Finite-time stabilization of uncertain triple integrator with only switch and gain, 37th Annual Conference on IEEE Industrial Electronics Society (IECON 2011), pp. 3942-3946, 2011

[17] T. Sanchez, J. Moreno, On a sign controller for the triple integrator, IEEE 52nd Annual Conference on Decision and Control (CDC), pp. 3566-3571, 2013.

[18] A. Bacciotti, L. Rosier, Lyapunov Functions and Stability in Control Theory, Springer, 237 p., 2005.

[19] V. Zubov, On systems of ordinary differential equations with generalized homogenous right-hand sides, Izvestia vuzov. Mathematica, vol. 1, pp. 80-88, 1958 (in Russian).

[20] V. I. Utkin, J. Guldner, J. Shi, Sliding Mode Control in ElectroMechanical Systems, CRC Press., 503 p., 2009.

[21] C. M. Dorling, A. S. I. Zinober, Two approaches to hyperplane design in multivariable variable structure control systems, International Journal of Control, vol. 44(1), pp. 65-82, 1986.

[22] F. L. Chernousko, I. M. Ananevskii, S. A. Reshmin, Control of nonlinear dynamical systems: methods and applications, Berlin: SpringerVerlag, 396 p., 2008

[23] A. Filippov, Differential equations with discontinuous right-hand sides, Dordrecht, Kluwer, 1988

[24] M. Tulio Angulo, J. Moreno, L. Fridman, Robust exact uniformly convergent arbitrary order differentiator, Automatica, vol. 49, pp. 2489-2495, 2013. 